\title{
Current Status of Indications, Timing, Management, Complications, and Outcomes of Tracheostomy in Traumatic Brain Injury Patients
}

\author{
Gabriel A. Quiñones-Ossa' \\ Y. A. Durango-Espinosa² \\ Luis Rafael Moscote-Salazar ${ }^{4}$ \\ S. Galwankar ${ }^{5} \quad$ J. Gerber ${ }^{5}$ \\ ${ }^{1}$ Faculty of Medicine, El Bosque University, Bogota, Colombia \\ ${ }^{2}$ Cartagena Neurotrauma Research Group Research Line, Faculty of \\ Medicine, University of Cartagena, Cartagena de Indias, Colombia \\ ${ }^{3}$ Center for Biomedical Research (CIB), Cartagena Neurotrauma \\ Research Group Research Line, Faculty of Medicine, University of \\ Cartagena, Cartagena de Indias, Colombia \\ ${ }^{4}$ Center for Biomedical Research (CIB), Faculty of Medicine - \\ University of Cartagena, Cartagena Colombia, CLaNi- Latin \\ American Council of Neurocritical Care, Cartagena, Colombia \\ 5 Department of Emergency Medicine, Sarasota Memorial Hospital, \\ Florida State University, Florida, United States \\ ${ }^{6}$ Department of Biochemistry, Medical College, Kolkata, India \\ ${ }^{7}$ Department of Community Medicine, MGM Medical College \& LSK \\ Hospital, Kishanganj, Bihar, India
}

\section{H. Padilla-Zambrano ${ }^{3}$ Jenny Ruiz ${ }^{2}$}

R. Holland III $^{5} \quad$ Amrita Ghosh ${ }^{6} \quad$ R. Pal ${ }^{7} \quad$ Amit Agrawal $^{8}$

${ }^{8}$ Department of Neurosurgery, All India Institute of Medical Sciences, Bhopal, Madhya Pradesh, India

Address for correspondence Amit Agrawal, MCh, Department of Neurosurgery, All India Institute of Medical Sciences, Saket Nagar, Bhopal 462020, Madhya Pradesh, India (e-mail: dramitagrawal@gmail.com).

\begin{abstract}
Keywords

- tracheostomy

- ICU

- traumatic brain injury

Tracheostomy is the commonest bedside surgical procedure performed on patients needing mechanical ventilation with traumatic brain injury (TBI). The researchers made an effort to organize a narrative review of the indications, timing, management, complications, and outcomes of tracheostomy in relation to neuronal and braininjured patients following TBI. The study observations were collated from the published literature, namely original articles, book chapters, case series, randomized studies, systematic reviews, and review articles. Information sorting was restricted to tracheostomy and its association with TBI. Care was taken to review the correlation of tracheostomy with clinical correlates including indications, scheduling, interventions, prognosis, and complications of the patients suffering from mild, moderate and severe TBls using Glasgow Coma Scale, Glasgow Outcome Scale, intraclass correlation coefficient, and other internationally acclaimed outcome scales. Tracheostomy is needed to overcome airway obstruction, prolonged respiratory failure and as indispensable component of mechanical ventilation due to diverse reasons in intensive care unit. Researchers are divided over early tracheostomy or late tracheostomy from days to weeks. The conventional classic surgical technique of tracheostomy has been superseded by percutaneous techniques by being less invasive with lesser complications, classified into early and late complications that may be life threatening. Additional studies have to be conducted to validate and streamline varied observations to frame evidence-based practice for successful weaning and decannulation. Tracheostomy is a safer option in critically ill TBI patients for which a universally accepted protocol for tracheostomy is needed that can help to optimize indications and outcomes.
\end{abstract}

License terms

() (1) $\ominus \circledast$ 


\section{Introduction}

Traumatic brain injury (TBI) is a global public health concern and one of the main causes of morbidity, disability, and mortality that has been associated as a risk factor for neurodegeneration and degenerative diseases. Globally, investigators from the clinical disciplines have expressed interest in the translational research on neuronal and brain-injured patients regarding respiratory management with special and increased interest on ventilator protection in the last few decades. ${ }^{1,2}$ Tracheostomy is the most common surgical procedure made in patients in intensive care unit (ICU) who require mechanical ventilation (MV) (6-20\%) ${ }^{3,4}$ The objective of this article was to make a narrative review of the tracheostomy relation in neuronal and brain-injured patients.

\section{Materials and Methods}

We attempted a comprehensive assembly of study reports by exploring various resources, published surveys, and field studies in which indications, timing, management, complications, and outcomes of tracheostomy in relation to neuronal and brain-injured patients following TBI were reported. Based on the search results, we identified 87 research publications from potentially relevant articles on tracheostomy in TBIs. The search terms included combinations of Medical Subject Headings (MeSH) terms and empirical taxonomies from tracheostomy in TBI from PubMed-Entrez, Cochrane library, Web of science, and the World Health Organization (WHO) global health library including searching of abstracts from scientific meetings. In the absence of monolithic pattern of reporting and nationally representative database, all the reports on tracheostomy in TBIs were considered in this review. First, study reports of tracheostomy in TBIs among research literature sources were sourced; second, reports containing indications, timing, management, complications impacting outcomes of TBIs were identified from all the possible sources; third, globally published information from apex bodies, for example, WHO, Center for Disease Control and Prevention, Atlanta United States, and others, were also sourced. Primary outcome variables included were indications, timing, management, complications impacting outcomes of TBIs

\section{Traumatic Brain Injury}

In the last few decades, TBI incidence has been steadily increasing especially in the rural areas of low- and middle-income countries due to the greater uses of motorized vehicles; therefore, it is expected that by 2020 TBI will be one of the major causes of death and disability. ${ }^{5-7}$ Due to prompt medical and surgical approaches, death has substantially come down in recent times, yet greater number of TBI victims are living with spectrum of sequelae and disabilities. ${ }^{8}$

\section{The Glasgow Coma Scale and TBI}

The Glasgow Coma Scale (GCS) is part of clinical practice guidelines conventionally used as bedside neurological scale in routine office practice since it was introduced in 1974. It helps the physician in the assessment of the level of consciousness in patients to determine severity of the TBI with suspected patients of TBI. GCS measures the eye opening ( 4 points), the verbal response ( 5 points), and the best motor response ( 6 points) for a total of minimum 3 to maximum 15 points. This score is associated with alterations in pathophysiological changes following TBI that is reflected in the total score; it will be from 13 to 15 (mild), 9 to 12 (moderate), and less than 8 (severe TBI), respectively..$^{910}$ Severe TBI with lowest score is related to unconsciousness and coma with corresponding higher complications; tracheostomy is part of the clinical practice guidelines in these patients of different research groups. ${ }^{11,12}$

\section{Tracheostomy and Its Indications in TBI}

The tracheostomy (or tracheotomy) is a hole made by a surgical procedure in the cervical neck and anterior trachea wall to provide an airway for the patient for its breathing without the normal airways (nose or mouth). This bedside procedure is done specially in patients who suffered trauma injuries or neurological disturbances, but may be used in other cases in the ICU.13,14 A prolonged period of tracheal intubation poses an increased risk of a wide range of complications, for example, ventilator-associated pneumonia, increased need for deep sedation, vocal cords damage, ventilator-associated sinusitis, and ventilator weaning delay among others. ${ }^{15} \mathrm{~A}$ prolonged intubation period gives us a standpoint in the decision tree toward an absolute indication for establishing a more stable surgical airway-tracheostomy this case scenario., ${ }^{3,16}$ There are three "categories" that indicate to realize of the procedure: airway obstruction may be due to trauma, airway infections or neoplasm, and even anaphylaxis (the objective is to establish a secure and useful airway in a patient with obstructed or inadequate airway). ${ }^{14,17}$

Tracheostomy is required in prolonged respiratory failure that may be caused by a concomitant disease such as chronic obstructive pulmonary disease, acute respiratory distress syndrome (ARDS) and fibrosis (the objective is to give the patient positive pressure ventilation to reduce the dead space and resistance in airway to facilitate the weaning process; also, this will reduce the sedative therapy requirements). ${ }^{14,17}$ Also, in MV patients and those with excessive secretions and impaired cough caused by neurological injuries or chronic infections, tracheostomy is required (the objective is to clear secretions to avoid them to get into the pulmonary tract).,14,17 Further, there are relative contraindications for tracheostomy procedure itself, namely in patients younger than 15 years, elderly patients, and patients with morbid obesity, with uncorrectable bleeding diathesis, having a gross distortion in the neck caused by a mass, hematoma, tumors, or scarring from other surgical interventions, having limited neck mobility or extension by cervical vertebral fusion, or those with any cervical spine instability or alteration. ${ }^{18,19}$ 


\section{Timing of Tracheostomy: Early versus Late}

As mentioned earlier, one of the objectives in the management of patients with TBI is the establishment of a permeable airway; in many cases, it is necessary to obtain a definitive airway with endotracheal intubation and MV. ${ }^{17}$ However, when the endotracheal intubation has to be prolonged beyond the expected time, lesions may appear in the laryngeal mucosa. This is the time when other types of access should be considered for a less harmful temporary or permanent airway. Wang et $\mathrm{al}^{20}$ had documented that tracheostomy has certain advantages over prolonged intubation. However, it is still debated whether the time of performing the procedure affects the clinically important outcomes. ${ }^{3}$ Further, there is a lack of consensus regarding early tracheostomy (ET) versus late tracheostomy (LT). Literature supports that these can go from one to several days, and the benefits regarding the incidence of pneumonia and mortality were variable, which makes evident the great heterogeneity in the different studies. ${ }^{21,22}$ Khalili et al defined ET as less than 6 days after admission and concluded that patients with ET had a significantly shorter hospital as well as ICU stay, although mortality rates were not significantly different between the two groups; also, the Glasgow Outcome Scale (GOS) more than 4 was higher and ventilator-associated pneumonia was lower among the ET group. They also showed that ET significantly improved prognosis at 6 months (GOS $>4$ ). Further, they observed that the scores of GCS and Rotterdam showed no effects on the 6-month prognosis. ${ }^{23}$ Two other studies also showed comparable results to the previous study-that patients with ET had a shorter ICU stay compared with LT patients,, ${ }^{20,22}$ fewer days of MV, shorter duration of hospital stay, and lower incidence of nosocomial pneumonia, ${ }^{20,22}$ though mortality rates in ET versus LT were comparable. ${ }^{22}$

Alali et al in a cohort of 1,811 patients also observed lower probabilities of pneumonia, deep vein thrombosis, and decubitus ulcers; hospital mortality was similar between groups; unlike the majority, they also evaluated other additional variables with increased possible benefits of ET. ${ }^{21}$ Two other large multicenter studies deserve to be mentioned. In one multicenter study called TracMan (tracheostomy management in critical care), 909 randomized patients underwent a tracheotomy after 1 to 4 days versus more than 10 days of ventilator assistance, and noted that timing of tracheostomy had no effect on mortality, stay in ICU, or hospital. However, they also observed that patient who underwent ET needed less sedation. ${ }^{14}$ Terragni et al in another multicenter study on 419 patients randomized to perform a tracheostomy after 6 to 8 days or 13 to 15 days of MV concluded that the time of the tracheostomy did not affect the incidence of ventilator-associated pneumonia and 28 days mortality. However, it reduced the duration of MV and ICU stay. ${ }^{24}$ These studies had certain limitations. The TracMan study studied patients with ET (1-4 days) of MV. The literature suggested that majority of the patients with MV during this period of time would be released from MV without the need for a tracheotomy; therefore, it is not clear how the early intervention arm for TracMan would be applied to most clinical settings. ${ }^{14}$
Terragni et al excluded patients with anatomical deformity of the neck, history of previous tracheotomy, chronic obstructive pulmonary disease, and pneumonia (with a higher percentage of tracheostomy performed in ICU) ${ }^{3,24}$ In spite of the above, these studies suggested that the tracheostomy is a safer option in critically ill patients, because no mortality or serious short-term complications were reported related to tracheostomy. ${ }^{14,24}$

Most studies define ET less than 5, 6, 8, or 10 days after admission to the definitive care centers. ${ }^{16,25}$ Based on the evidence from available literature, it is suggested that placement of the tracheostomy can be deferred for at least 2 weeks to ensure the need for ventilator support following acute respiratory failure. All of these studies ${ }^{20,25,26}$ were inclined toward the reduction in the stay in the ICU, MV, and hospital stay. Pneumonia associated with MV can also be reduced with ET according to the literature, but mortality is usually quite comparable between both groups. The heterogeneity in the population of these studies, the variation in the quality of the study, and the lack of direct care protocol make it difficult to draw conclusions about clinical outcomes between ET and LT; however, majority of the studies have demonstrated a beneficial effect ET, with minimal harmful effects. ${ }^{16}$

\section{Percutaneous Dilatational and Surgical Tracheostomy}

Currently, there are two techniques used to perform tracheostomy; however, still there is a need to decide which would be better. The first is the conventional surgical tracheostomy (ST) that is the classic technique of performing a tracheostomy until the next generation or percutaneous dilatational tracheostomy (PDT) arrived. ${ }^{27}$ Several clinical studies, meta-analysis, systematic reviews, and secondary data analysis have compared PDT and ST, suggesting equivalences and even the potential superiority of PDT over ST. ${ }^{28-32}$ Two meta-analysis and one systematic review showed a statistically significant reduction in stoma infection and operative time with PDT. ${ }^{28,29,31}$ In addition, one of the two meta-analysis showed that PT techniques reduced the chances of major postoperative bleeding. ${ }^{31}$ However, two recent systematic reviews showed no statistically significant differences with respect to postoperative bleeding rates, ${ }^{29}$ surgical bleeding, and mortality. ${ }^{29,30}$ The longitudinal follow-up suggested that the delayed complications, which included clinically significant tracheal stenosis, were similar compared with these techniques. ${ }^{3,33}$ PDT has gained wide acceptance, due to its great ease, for the advantages that have been observed in several studies, so it has become the predominant method of tracheotomy placement. ${ }^{3,34,35}$

\section{Management of TBI Patients with Tracheostomy}

As mentioned previously in this review, one of the main objectives in patients with a GCS less than 8 is to establish MV to prevent hypoxemia and hypercapnia that may occur as a second insult in severe TBI cases. ${ }^{1}$ It is well known 
that in medical practice, the prophylactic hyperventilation for prolonged periods should be avoided; but it is unclear if tidal volume and the positive end-expiratory pressure (PEEP) should have an exact value for the patients with TBI and those undergoing tracheostomy management in the ICU. Even though, some studies have shown evidence that a higher tidal volume and lower PEEP were presented with neurological injury; also a prolonged MV, nosocomial infections, and mortality rates are higher in this patients. ${ }^{36,37}$ The management inside the ICU of becomes complex because it is related to the maintenance of a good ventilation, weaning, and extubation issues. ${ }^{38}$

Severe TBI with impaired consciousness due to brain stem injury with subsequent hypoventilation (that produces the secondary brain insults) and the related complications has bad outcomes. ${ }^{39}$ Because of this, it is recommended that once the GCS is established with less than 8 scores, the practitioner must perform an endotracheal intubation ${ }^{40}$ the main objective is to increase the inspired oxygen fraction higher than $60 \mathrm{~mm} \mathrm{Hg}$ to avoid ischemia of brain tissues; even though, if it is diagnosed, this value can be modified and scrutinized by monitoring of low tissue oxygen tension and the implementation of a low jugular venous $\mathrm{O}_{2}$ saturation ${ }^{40}$. Also, the partial pressure of $\mathrm{CO}_{2}(\mathrm{PaCO} 2)$ should be measured to determine the cerebral blood flow that is related to the intracranial pressure (ICP); if the physician controls $\mathrm{PaCO}_{2}$ and keeps the levels between 32 and $45 \mathrm{~mm} \mathrm{Hg}$ in TBI cases, it shows evidence of better prognosis. ${ }^{38,40,41}$ Even now, there is no consensus on the values of tidal volume to get a $\mathrm{PaCO}_{2}$ into the recommended range. ${ }^{40}$ Special attention is needed in TBI patients from increased risk of ventilator-induced lung injury and ARDS due to high tidal volumes leading to worse prognosis; low tidal volume is recommended in the braininjured patients to avoid these complication. ${ }^{40-43}$ Another issue is the control of PEEP as with its increase it produces a higher intrathoracic pressure impairing the central venous return to increase ICP; it is recommended to use less than $5 \mathrm{~cm} \mathrm{H}_{2} \mathrm{O}$ of PEEP values. ${ }^{37,40,42}$ Some studies have shown that a PEEP increased to $15 \mathrm{~cm} \mathrm{H}_{2} \mathrm{O}$ does not produce significant changes in the ICP. In brain tissue oxygenation, higher levels lead to a better oxygenation, thus a PEEP increment is safe in brain-injured patients with ARDS without hemodynamic alteration to improve the prognosis and outcomes. ${ }^{2,40,44}$

\section{Pediatric Perspective}

As in adults, the tracheostomy procedure in pediatrics should be considered if the patients fulfill all the criteria mentioned above, especially if there is an inability to maintain a secure airway, the prolonged ventilation, or even the airway obstruction ${ }^{45}$ for this population the tracheostomy timing is between 7 and 10 days. ${ }^{23}$ In the pediatric population, the TBI is a major cause of death and disabilities in patients from 1 to 19 years old, ${ }^{45,46}$ commonly caused by falls in the youngest patients ( $<2$ years), while the traffic road motor vehicle accident is the common cause in adolescents. ${ }^{47}$ At birth, general requirements are lower and increase at its maximum levels at
9 years of age approximately. ${ }^{48}$ Therefore, children require less air volume than adults even when they have higher oxygen requirements. ${ }^{49}$ After a severe injury is important to establish a good airway management, the tracheostomy in the children allows them an early transition from the ICU to the natural home environment. ${ }^{50}$ The main objective in the pediatric critical care is to maintain an appropriate CPP with an ICP less than $20 \mathrm{~mm} \mathrm{Hg.}{ }^{45}$ Sometimes the increase in ICP can be treated with therapeutic hyperventilation, but this treatment can lead to a $\mathrm{PCO}_{2}$ decrease that in extreme levels decrease the cerebral perfusion (this is because the blood flow in these patients is sensitive to the $\mathrm{CO}_{2}$ serum levels). ${ }^{48}$ Further, the brain ischemia, due to the hyperventilation and subsequent hypocapnia, decrease the $\mathrm{HCO}_{3}$ levels leading to the decreased oxygen release in the brain. This is one of the main problems with the hyperventilation management that forces the treating physicians to consider it as a short-term treatment to reduce the ICP. ${ }^{50}$

\section{Complications}

As in most of the medical and surgical procedures, complications following tracheostomy are classified into two types: early and late complications; these may also be life-threatening ( - Table 1) $)^{51-56}$ Early complications occur within the first week, namely bleeding that starts during the procedure and in less than 48 hours is common due to the injury; but later it can be harmful for the patient's life by the tracheostomy tube or airway occlusion. ${ }^{54,57-59}$ Tube obstruction as mentioned before may be caused by a blood clot, or also by mucous plugs, angulation of the tube, or even a false lumen. Infections may be caused due to the bad wound care and also nosocomial pneumonia. Tube dislodgement occurs within the first week and is a medical emergency because it could be difficult to replace creating a false tract; it occurs when the patient is moved, or the tube is replaced, or even when the patients by itself get extubated. ${ }^{22,54,57-60}$ The late complications appear with a prolonged tube placement and are divided by the anatomic location related to the stoma (suprastomal, stomal, and infrastomal) ${ }^{55,57}$ When the patients undergo cricothyroidotomy, the suprastomal complications are the most frequent

Table 1 Complications of tracheostomy

\begin{tabular}{|l|}
\hline Pneumothorax \\
\hline Hemorrhage \\
\hline Tube/cannula misplacement \\
\hline Esophageal injury and tracheoesophageal fistula \\
\hline Thyroid injury \\
\hline Recurrent laryngeal nerve injury \\
\hline Subcutaneous emphysema \\
\hline Cardiopulmonary arrest/mortality \\
\hline Infection \\
\hline Tracheal stenosis \\
\hline Tracheomalacia \\
\hline Voice change \\
\hline
\end{tabular}


injuries and the patients present with tracheal or subglottic stenosis. ${ }^{55,57}$ Stomal complications are caused by the injury of the anterior tracheal wall; this one invaginates protruding in the lumen causing a tracheal obstruction resulting in the stomal stenosis. The infrastomal complications are the stenosis, the tracheomalacia, and the fistulas (tracheoesophageal and tracheoinnominate), as well as ischemia of the walls due to the prolonged pressure of the tube; this latter one may lead to tracheal ulceration and posterior tracheal necrosis. ${ }^{55,57}$

\section{Weaning from Ventilator}

The guidelines for weaning from ventilator were developed 10 years ago, ${ }^{1}$ which required the patient to meet adequate criteria. ${ }^{61}$ However, traditional weaning parameters are not good predictors of a successful outcome in neurocritical care; for this reason, these parameters are not reliable in neuroemergencies. ${ }^{62}$ In a multicenter observational study, ${ }^{37}$ which included patients with TBI, stroke, and subarachnoid hemorrhage, showed that the duration of MV is greater, compared with other groups of patients in the ICU presenting greater challenges to the clinician for weaning in the neurological patients. There is very little evidence for the patient with TBI regarding this topic, in fact, the failure rate of extubation is up to $38 \%{ }^{63}$ and the rates of delayed extubation are also high. ${ }^{1}$ It is recommended that weaning in these patients should be initiated only after the improvement in the pathology and careful consideration of the risks and benefits of continuing ventilator support. ${ }^{64} \mathrm{~A}$ multicenter study on 437 patients evaluated four characteristics-namely, ageless than 40 years, visual pursuit, attempts to swallow, and GCS score more than 10-that were associated with successful extubation in patients with severe $\mathrm{TBI}^{65}$. Each characteristic counted as a point, and a score 3 or more was associated with $90 \%$ extubation success. Another multicentric observational study on 192 patients evaluated elements other than GCS, associated with the success of extubation, which included juvenile age, presence of cough, and negative fluid balance. ${ }^{66}$ Despite these studies that create scores to predict success, they lack external validation making generalization of these results difficult, which is why studies are still needed to confirm the applicability of these to the patients with TBI.

\section{Decannulation Protocol}

Decannulation involves the valuation of multiple variables, and although this is a process with little scientific evidence and that requires interdisciplinary coordination, its arduous work could reduce the time of use of the tracheostomy, accelerate weaning, and increase safety for the patient while decreasing the risk of failure and complications ${ }^{67}$ In this context, decannulation is usually based on particular scenarios in each institution, adding the clinical condition of the patients and the experience of the treating team. Like the weaning of ventilation, it is very difficult to predict the success or not of decannulation, and the proper time to perform the procedure is unknown. For this reason, numerous studies have been performed that attempt to introduce predictive criteria for successful decannulation..$^{68}$

A multicenter study on 831 patients, of which 470 were decannulated, associated measurable parameters at admission such as an oral diet and a higher alert status as predictors of successful decannulation. ${ }^{69}$ In addition, they found a negative association with age, duration of MV, and complications. Enrichi et al, in a population of 74 patients with acquired brain injury, found that the criteria that showed the highest values of sensitivity and specificity for the prediction of successful decannulation were plugging the tracheostomy tube (80-100\%), endoscopic airway patency evaluation (100-30\%), swallowing assessment (85-96\%), and blue dye test (65-85\%). ${ }^{69,70}$ All these criteria that were combined into a single clinical parameter had greater sensitivity (100\%) than specificity (82\%). In addition, they evaluated other parameters such as voluntary cough, cough reflex, tracheal suction numbers, oxygen saturation, and the level of consciousness. ${ }^{69,70}$

A systematic review evaluated predictors of successful decannulation and proposed a predictive score, the results of which are similar to the studies mentioned above. They divided the parameters into two groups: quantitative objectives and semiquantitative parameters. ${ }^{71}$ The review established that-if all the main criteria are counted, independently of the minor criteria-in decannulation, there is a probability of positive results if the two major criteria are met: (1) a careful evaluation is needed and (2) if most minor criteria are met. ${ }^{71}$ It is important to remember that it is a hypothetical score and requires validation in daily practice. Two recent studies, a systematic review and a retrospective study, associated positive spontaneous cough in patients with TBI as a predictor of the success of decannulation, ${ }^{72,73}$ a result that is consistent with the literature reviewed. In general, decannulation is more often individualized than protocol based. In this sense, randomized controlled studies can do it more based on evidence.

\section{Outcome}

TBI may present, related to the severity of the injury, a tracheostomy due to the bad airway functionality. Due to this, many patients will have a prolonged MV as one of the main outcomes secondary to this entity. Sometimes patients will present with a prolonged in-hospital or ICU stay due to the bad health status and comorbidities, as well as with nosocomial infections or thrombosis/embolism events due to the lack of movement that may also produce pressure ulcers in the patients, especially in those that have a GCS less than 8 points, have neurological degenerative disorder, or are elder. ${ }^{21}$ It has been noted that age is related to the prognosis outcome after a TBI; younger patients have higher recovery rates. In 2014, a study showed that for elderly population the injury related to GCS had a higher mortality rate than younger population (under 55) (i.e., GCS as a better death predictor); younger patients with worst injuries than the elderly endured a better outcome. ${ }^{74}$ Knowing this, it can be 
explained why in the aged population, there is a decreased likelihood to realize a tracheostomy, by taking into account the outcome prognosis and the possible comorbidities and functional baseline deterioration compared with the younger patients. However, in a study on the general population in 2017, it was found that patients with severe TBI and those that did not underwent tracheostomy had a shorter ICU stay and less MV days (this reduces the risk of nosocomial pneumonia and further complications). Current medical practice envisages that every patient has unique and individual requirements, and hence it is better not to generalize but to treat according to a tailor-made approach. ${ }^{75}$

\section{Conclusions}

TBI incidence has been steadily increasing globally and tracheostomy has been accepted as an integral intervention in critically ill patients depending on risk correlates. Tracheostomy is accepted as noninvasive lifesaving bedside procedure for which a comprehensively unanimous clinical practice guideline is the call of the day to optimize indication to outcomes. Further, controlling complications including nosocomial infections is inherent challenge. In the era of patient centered evidence based medical care, we need to move forward with futuristic vision for lower complications, higher success rates combined with optimal weaning, and decannulation taking caregiver in confidence.

\section{Conflict of Interest}

None declared.

\section{References}

1 Asehnoune K, Roquilly A, Cinotti R. Respiratory management in patients with severe brain injury. Crit Care 2018;22(1):76

2 Asehnoune K, Mrozek S, Perrigault PF, et al; BI-VILI study group. A multi-faceted strategy to reduce ventilation-associated mortality in brain-injured patients. The BI-VILI project: a nationwide quality improvement project. Intensive Care Med 2017;43(7):957-970

3 Freeman BD. Tracheostomy update: when and how. Crit Care Clin 2017;33(2):311-322

4 Mehta AB, Syeda SN, Bajpayee L, Cooke CR, Walkey AJ, Wiener RS. Trends in tracheostomy for mechanically ventilated patients in the United States, 1993-2012. Am J Respir Crit Care Med 2015;192(4):446-454

5 Agoston DV, Shutes-David A, Peskind ER. Biofluid biomarkers of traumatic brain injury. Brain Inj 2017;31(9):1195-1203

6 Byass P, de Courten M, Graham WJ, et al. Reflections on the global burden of disease 2010 estimates. PLoS Med 2013;10(7):e1001477

7 Galgano M, Toshkezi G, Qiu X, Russell T, Chin L, Zhao LR. Traumatic brain injury: current treatment strategies and future endeavors. Cell Transplant 2017;26(7):1118-1130

8 DeKosky ST, Asken BM. Injury cascades in TBI-related neurodegeneration. Brain Inj 2017;31(9):1177-1182

9 Papa L, Edwards D, Ramia M. Exploring serum biomarkers for mild traumatic brain injury. In: Kobeissy FH, editor. Brain Neurotrauma: Molecular, Neuropsychological, and Rehabilitation Aspects; 2015. Boca Raton (FL): CRC Press/ Taylor \& Francis. Chapter 22. Available at: https://www.ncbi. nlm.nih.gov/books/NBK299199/
10 Werhane ML, Evangelista ND, Clark AL, et al. Pathological vascular and inflammatory biomarkers of acute- and chronic-phase traumatic brain injury. Concussion 2017;2(1):CNC30

11 Reith FC, Van den Brande R, Synnot A, Gruen R, Maas AI. The reliability of the Glasgow Coma Scale: a systematic review. Intensive Care Med 2016;42(1):3-15

12 Teasdale G, Jennett B. Assessment of coma and impaired consciousness. A practical scale. Lancet 1974;2(7872) :81-84

13 Mitchell RB, Hussey HM, Setzen G, et al. Clinical consensus statement: tracheostomy care. Otolaryngol Head Neck Surg 2013;148(1):6-20

14 Young D, Harrison DA, Cuthbertson BH, Rowan K; TracMan Collaborators. Effect of early vs late tracheostomy placement on survival in patients receiving mechanical ventilation: the TracMan randomized trial. JAMA 2013;309(20):2121-2129

15 Young SR, Bouloux GF, Perez SD, Abramowicz S. Does length of intubation before tracheostomy affect intensive care unit length of stay? Oral Surg Oral Med Oral Pathol Oral Radiol 2017;124(6):525-528

16 Cheung NH, Napolitano LM. Tracheostomy: epidemiology, indications, timing, technique, and outcomes. Respir Care 2014;59(6):895-915, discussion 916-919

17 Doyle JD, Scales DC. Tracheostomy. Principles of Critical Care. New York, NY: McGraw-Hill; 2015

18 El Solh AA, Jaafar W. A comparative study of the complications of surgical tracheostomy in morbidly obese critically ill patients. Crit Care 2007;11(1):R3

19 Warren WH. Percutaneous dilatational tracheostomy: a note of caution. Crit Care Med 2000;28(5):1664-1665

20 Wang HK, Lu K, Liliang PC, et al. The impact of tracheostomy timing in patients with severe head injury: an observational cohort study. Injury 2012;43(9):1432-1436

21 Alali AS, Scales DC, Fowler RA, et al. Tracheostomy timing in traumatic brain injury: a propensity-matched cohort study. J Trauma Acute Care Surg 2014;76(1):70-76, discussion 76-78

22 Zirpe KG, Tambe DV, Deshmukh AM, Gurav SK. The impact of early tracheostomy in neurotrauma patients: a retrospective study. Indian J Crit Care Med 2017;21(1):6-10

23 Khalili H, Paydar S, Safari R, Arasteh P, Niakan A, Abolhasani Foroughi A. Experience with traumatic brain injury: is early tracheostomy associated with better prognosis? World Neurosurg 2017;103:88-93

24 Terragni PP, Antonelli M, Fumagalli R, et al. Early vs late tracheotomy for prevention of pneumonia in mechanically ventilated adult ICU patients: a randomized controlled trial. JAMA 2010;303(15):1483-1489

25 Dunham CM, Cutrona AF, Gruber BS, Calderon JE, Ransom KJ, Flowers LL. Early tracheostomy in severe traumatic brain injury: evidence for decreased mechanical ventilation and increased hospital mortality. Int J Burns Trauma 2014;4(1):14-24

26 Paydar S, Khalili H, Mousavi SM. Perfect timing of tracheostomy in patients with traumatic brain injury. Bull Emerg Trauma 2014;2(3):136-137

27 Timbrell D, Jankowski S. Management of and indications for tracheostomy in care of the critically ill patient. Surgery 2018;36:187-195

28 Delaney A, Bagshaw SM, Nalos M. Percutaneous dilatational tracheostomy versus surgical tracheostomy in critically ill patients: a systematic review and meta-analysis. Crit Care 2006;10(2):R55

29 Johnson-Obaseki S, Veljkovic A, Javidnia H. Complication rates of open surgical versus percutaneous tracheostomy in critically ill patients. Laryngoscope 2016;126(11):2459-2467

30 Klotz R, Probst P, Deininger M, et al. Percutaneous versus surgical strategy for tracheostomy: a systematic review and meta-analysis of perioperative and postoperative complications. Langenbecks Arch Surg 2018;403(2):137-149 
31 Putensen C, Theuerkauf N, Guenther U, Vargas M, Pelosi P. Percutaneous and surgical tracheostomy in critically ill adult patients: a meta-analysis. Crit Care 2014;18(6):544

32 Vargas M, Buonanno P, Giorgiano L, Sorriento G, Iacovazzo C, Servillo G. Comparison between surgical and percutaneous tracheostomy effects on procalcitonin kinetics in critically ill patients. Crit Care 2018;22(1):297

33 Dempsey GA, Morton B, Hammell C. Williams LT, Tudur Smith C, Jones T. Long-Term outcome following tracheostomy in critical care: a systematic review. Crit Care Med 2016;44(3):617-628

34 Akulian JA, Yarmus L, Feller-Kopman D. The role of cricothyrotomy, tracheostomy, and percutaneous tracheostomy in airway management. Anesthesiol Clin 2015;33(2):357-367

35 Kidane B, Pierre AF. From open to bedside percutaneous tracheostomy. Thorac Surg Clin 2018;28(3):263-276

36 Lepelletier D, Roquilly A, Demeure dit latte D, et al. Retrospective analysis of the risk factors and pathogens associated with early-onset ventilator-associated pneumonia in surgical-ICU head-trauma patients. J Neurosurg Anesthesiol 2010;22(1):32-37

37 Pelosi P, Ferguson ND, Frutos-Vivar F, et al; Ventila Study Group. Management and outcome of mechanically ventilated neurologic patients. Crit Care Med 2011;39(6):1482-1492

38 Longhi L, Pagan F, Valeriani V, et al. Monitoring brain tissue oxygen tension in brain-injured patients reveals hypoxic episodes in normal-appearing and in peri-focal tissue. Intensive Care Med 2007;33(12):2136-2142

39 Rosenfeld JV, Maas AI, Bragge P, Morganti-Kossmann MC, Manley GT, Gruen RL. Early management of severe traumatic brain injury. Lancet 2012;380(9847) :1088-1098

40 Carney N, Totten AM, O'Reilly C, et al. Guidelines for the management of severe traumatic brain injury, Fourth Edition. Neurosurgery 2017;80(1):6-15

41 Davis DP, Idris AH, Sise MJ, et al. Early ventilation and outcome in patients with moderate to severe traumatic brain injury. Crit Care Med 2006;34(4):1202-1208

42 Gattinoni L, Protti A, Caironi P, Carlesso E. Ventilator-induced lung injury: the anatomical and physiological framework. Crit Care Med 2010;38(10, Suppl):S539-S548

43 Mascia L, Zavala E, Bosma K, et al; Brain IT group. High tidal volume is associated with the development of acute lung injury after severe brain injury: an international observational study. Crit Care Med 2007;35(8):1815-1820

44 Nemer SN, Caldeira JB, Santos RG, et al. Effects of positive end-expiratory pressure on brain tissue oxygen pressure of severe traumatic brain injury patients with acute respiratory distress syndrome: a pilot study. J Crit Care 2015;30(6):1263-1266

45 Atike Ongun E, Dursun O. Prediction of mortality in pediatric traumatic brain injury: implementations from a tertiary pediatric intensive care facility. Ulus Travma Acil Cerrahi Derg 2018;24(3):199-206

46 Mtaweh $\mathrm{H}$, Bell MJ. Management of pediatric traumatic brain injury. Curr Treat Options Neurol 2015;17(5):348

47 Duhaime AC, Alario AJ, Lewander WJ, et al. Head injury in very young children: mechanisms, injury types, and ophthalmologic findings in 100 hospitalized patients younger than 2 years of age. Pediatrics 1992;90(2 Pt 1) :179-185

48 Udomphorn Y, Armstead WM, Vavilala MS. Cerebral blood flow and autoregulation after pediatric traumatic brain injury. Pediatr Neurol 2008;38(4):225-234

49 McFadyen JG, Ramaiah R, Bhananker SM. Initial assessment and management of pediatric trauma patients. Int J Crit Illn Inj Sci 2012;2(3):121-127
50 Can FK, Anıl AB, Anıl M, et al. The outcomes of children with tracheostomy in a tertiary care pediatric intensive care unit in Turkey. Turk Pediatri Ars 2018;53(3):177-184

51 Kumar VAK, Kiran NAS, Kumar VA, et al. The outcome analysis and complication rates of tracheostomy tube insertion in critically ill neurosurgical patients; a data mining study. Bull Emerg Trauma 2019;7(4):355-360

52 Kumar VA, Reddy BU, Kiran Kumar VA, et al. Speech and swallowing function outcome following early tracheostomy in patients who underwent neurosurgical intervention. Indian J Crit Care Med 2018;22(6):427-430

53 Cipriano A, Mao ML, Hon HH, et al. An overview of complications associated with open and percutaneous tracheostomy procedures. Int J Crit Illn Inj Sci 2015;5(3):179-188

54 Durbin CG Jr. Early complications of tracheostomy. Respir Care 2005;50(4):511-515

55 Epstein SK. Late complications of tracheostomy. Respir Care 2005;50(4):542-549

56 Griffiths J, Barber VS, Morgan L, Young JD. Systematic review and meta-analysis of studies of the timing of tracheostomy in adult patients undergoing artificial ventilation. BMJ 2005;330(7502):1243

57 Fernandez-Bussy S, Mahajan B, Folch E, Caviedes I, Guerrero J, Majid A. Tracheostomy tube placement: early and late complications. J Bronchology Interv Pulmonol 2015;22(4):357-364

58 Pal P, Sood AS, Singla S. Early complications of tracheostomy: a study on 100 patients at a single tertiary care centre. International Journal of Otorhinolaryngology and Head and Neck Surgery 2017;4:217-221

59 Panajaroen P, Tangjaturonrasme N. Pneumothorax after tracheostomy: a prospective study. Otolaryngol Pol 2015; 69(3):26-30

60 Heffner JE, Miller KS, Sahn SA. Tracheostomy in the intensive care unit. Part 2: complications. Chest 1986;90(3):430-436

61 Jackson M, Strang T, Rajalingam Y. A practical approach to the difficult-to-wean patient. J Intensive Care Soc 2012;13:327-331

62 Ko R, Ramos L, Chalela JA. Conventional weaning parameters do not predict extubation failure in neurocritical care patients. Neurocrit Care 2009;10(3):269-273

63 Cinotti R, Bouras M, Roquilly A, Asehnoune K. Management and weaning from mechanical ventilation in neurologic patients. Ann Transl Med 2018;6(19):381

64 Swain A, Bhagat H, Sahni N, Salunke P. Mechanical ventilation in neurological and neurosurgical patients. Neurol India 2016;64(3):485-493

65 Asehnoune K, Seguin P, Lasocki S, et al; ATLANREA group. Extubation success prediction in a multicentric cohort of patients with severe brain injury. Anesthesiology 2017; 127(2):338-346

66 McCredie VA, Ferguson ND, Pinto RL, et al; Canadian Critical Care Trials Group. Airway management strategies for brain-injured patients meeting standard criteria to consider extubation. a prospective cohort study. Ann Am Thorac Soc 2017;14(1):85-93

67 Zanata IdeL, Santos RS, Hirata GC. Tracheal decannulation protocol in patients affected by traumatic brain injury. Int Arch Otorhinolaryngol 2014;18(2):108-114

68 O'Connor HH, White AC. Tracheostomy decannulation. Respir Care 2010;55(8):1076-1081

69 Heidler MD, Salzwedel A, Jöbges M, et al. Decannulation of tracheotomized patients after long-term mechanical ventilation - results of a prospective multicentric study in German neurological early rehabilitation hospitals. BMC Anesthesiol 2018;18(1):65 
70 Enrichi C, Battel I, Zanetti C, et al. Clinical criteria for tracheostomy decannulation in subjects with acquired brain injury. Respir Care 2017;62(10):1255-1263

71 Santus P, Gramegna A, Radovanovic D, et al. A systematic review on tracheostomy decannulation: a proposal of a quantitative semiquantitative clinical score. BMC Pulm Med 2014;14:201

72 Perin C, Meroni R, Rega V, Braghetto G, Cerri CG. Parameters influencing tracheostomy decannulation in patients undergoing rehabilitation after severe acquired brain injury (SABI) Int Arch Otorhinolaryngol 2017;21(4):382-389
73 Singh RK, Saran S, Baronia AK. The practice of tracheostomy decannulation-a systematic review. J Intensive Care 2017;5:38

74 Salottolo K, Levy AS, Slone DS, Mains CW, Bar-Or D. The effect of age on Glasgow Coma Scale score in patients with traumatic brain injury. JAMA Surg 2014;149(7):727-734

75 Humble SS, Wilson LD, McKenna JW, et al. Tracheostomy risk factors and outcomes after severe traumatic brain injury. Brain Inj 2016;30(13-14):1642-1647 